\title{
Harmful neutrophil subsets in patients with ischemic stroke
}

\author{
Association with disease severity
}

David Weisenburger-Lile, MD, Yuan Dong, PhD, Marion Yger, MD, Gaëlle Weisenburger, MD,

Giulia Frasca Polara, MD, Thomas Chaigneau, MS, Riccardo Zapata Ochoa, MS, Beatrice Marro, MD,

Bertrand Lapergue, MD, PhD, Sonia Alamowitch, MD, and Carole Elbim, MD, PhD

\author{
Correspondence \\ Dr. Carole Elbim \\ carole.elbim@upmc.fr
}

\section{Abstract \\ Objective \\ To better understand the functional state of circulating neutrophils in patients with ischemic stroke (IS) for planning future clinical trials. \\ Methods \\ We analyzed by flow cytometry activation state of circulating neutrophils and the distribution of neutrophil peripheral subsets in 41 patients with acute IS less than 6 hours before admission and} compared them with 22 age-matched healthy controls.

\section{Results}

Our results demonstrated continuous basal hyperactivation of circulating neutrophils during acute IS, characterized by lower L-selectin expression and higher CD11b expression at the cell surface, increased ROS production by neutrophils, and greater circulating levels of neutrophil elastase. Neutrophil hyperactivation was associated with deregulation of the equilibrium between apoptotic and necrotic. Patients also had higher percentages than controls of the overactive senescent $\left(\mathrm{CXCR} 4^{\text {bright }} / \mathrm{CD} 62 \mathrm{~L}^{\mathrm{dim}}\right)$ neutrophil subset and increased percentage of neutrophils with a reverse transendothelial migration ( $\left.\mathrm{CD} 54{ }^{\text {high }} \mathrm{CXCR} 1^{\text {low }}\right)$ phenotype. Importantly, neutrophil alterations were associated with the clinical severity of the stroke, evaluated by its NIH Stroke Scale score.

\section{Conclusion}

Altogether, our results indicate that during acute IS, the inflammatory properties of circulating neutrophils rise, associated with the expansion of harmful neutrophil subsets. These changes in neutrophil homeostasis, associated with disease severity, may play an instrumental role by contributing to systemic inflammation and to the blood-brain barrier breakdown. Our findings highlight new potential therapeutic approaches of stroke by rebalancing the ratio of senescent to immunosuppressive neutrophils or decreasing reverse neutrophil transmigration or both.

From the Sorbonne Universités (D.W.-L., Y.D., T.C., R.Z.O., S.A., C.E.), UPMC Univ Paris 06, UMRS 938, CdR Saint-Antoine, Hôpital Saint-Antoine; INSERM (D.W.-L., Y.D., T.C., R.Z.O., S.A. C.E.), UMRS 938, CdR Saint- Antoine, Team "Immune System, Neuroinflammation and Neurodegenerative Diseases," Hôpital St-Antoine; Service de Neurologie et d'Urgences Neurovasculaires (D.W.-L., M.Y., S.A.), Assistance Publique-Hôpitaux de Paris, Hôpital Saint-Antoine; Division of Pneumology (G.W.), Foch Hospital, F-92150, Suresnes; Division of Neurology (G.F.P.), Stroke Center, Assistance Publique-Hôpitaux de Paris, Pitié-Salpêtrière Hospital; Division of Radiology (B.M.), Assistance Publique-Hôpitaux de Paris, Hôpital SaintAntoine; and Division of Neurology (B.L.), Stroke Center, Foch Hospital, F-92150, Suresnes. 


\section{Glossary}

AAD = amino-actinomycin D; ANOVA = analysis of variance; $\mathbf{A P C}=$ allophycocyanine $\mathbf{B B B}=$ blood-brain barrier; $\mathbf{D A M P}=$ danger-associated molecular pattern; $\mathbf{f M L P}=\mathrm{N}$-formylmethionyl-leucyl-phenylalanine; $\mathrm{HC}=$ healthy control; $\mathrm{HE}=$ hydroethidine; HMGB = high-mobility group box; IS = ischemic stroke; LPS = lipopolysaccharide; $\mathbf{M M P}=$ matrix metalloproteinase; $\mathbf{M P O}=$ myeloperoxidase; NET = neutrophil extracellular trap; NIHSS = NIH Stroke Scale; NLR = Nod-like receptor; PBS = phosphate buffered saline; PMN = polymorphonuclear neutrophil; $\mathbf{R O S}=$ reactive oxygen species; $\mathbf{r T E M}=$ reverse transendothelial migration; sJAM-C = soluble JAM-C; TNF = tumor necrosis factor; TLR $=$ Toll-like receptor.

Cerebral ischemia elicits a strong inflammatory response involving the transmigration of several subsets of leukocytes, including neutrophils. ${ }^{1}$ Murine models of ischemic stroke (IS) reveal that neutrophils are the first cells recruited into the brain, minutes after stroke, accompanied by local production of cytokines or chemokines and facilitated by a very early ischemia-induced breakdown in the blood-brain barrier (BBB). ${ }^{2,3}$ Massive infiltration of neutrophils into ischemic brain tissue has also been documented in human stroke patients. ${ }^{4}$ Although animal stroke models suggest that depleting circulating neutrophils before or at the onset of stroke reduces infarct size, human drug trials aimed at preventing potentially harmful subacute neutrophil adhesion and infiltration into the brain parenchyma poststroke have not improved patient recovery. ${ }^{5,6}$ The failure of these trials might be partially explained by the undiscriminating nature of these approaches: the anti-inflammatory potential of some circulating neutrophil subsets means that globally blocking neutrophil access to the brain might not be a viable therapeutic approach. A better understanding of the functional state of circulating neutrophils in patients with IS is therefore crucial for planning future clinical trials targeting postischemic inflammation.

Neutrophils, the most abundant circulating leukocytes, play a key role in the immune defense against bacterial and fungal pathogens. They are rapidly recruited from blood to sites of infection, where they kill microorganisms via a number of different mechanisms including phagocytosis, production of reactive oxygen species (ROS), release of granular proteins, neutrophil extracellular traps (NETs), a process called NETosis. However, in case of inappropriate and/or excessive activation, neutrophils play also a key role in the induction or promotion of inflammation-induced tissue injury. ${ }^{7}$ Recent studies point to the existence of heterogenous populations of mature and immature neutrophils. In fact, diverse subsets of neutrophils differ markedly in their proinflammatory activity. Senescent neutrophils, an overly active subset, express elevated levels of CXCR4, ${ }^{8}$ whereas the immunosuppressive $\mathrm{CD} 16^{\text {bright }} / \mathrm{CD} 62 \mathrm{~L}^{\text {dim }}$ subset exhibits reduced proinflammatory properties. ${ }^{9}$ Recent studies also demonstrate that neutrophils can migrate back into the circulation, in a process referred to as reverse transendothelial migration (rTEM). ${ }^{10}$

Contradictory results on the phenotype and functions of circulating neutrophils in patients with IS have been reported. Some authors report fewer oxidative bursts and NET production, ${ }^{11}$ whereas others suggest increased degranulation and ROS production. $^{12}$ Some analyzed neutrophil functions $24-48$ hours after IS, although it is now clear that inflammatory pathways are involved in the first hours. ${ }^{1}$ None has investigated the distribution of the senescent, immunosuppressive, and rTEM neutrophil subsets or their consequences on the course of IS.

Our study, performed in whole blood to avoid artifacts due to isolation procedures, ${ }^{13}$ aimed to characterize fully the phenotypes and functions of human peripheral neutrophils at different stages of acute IS. We analyzed neutrophil activation state and the distribution of 3 neutrophil subsets, the senescent subset, the immunosuppressive subset, and the rTEM subset in the aim of addressing the right targets for planning future clinical trials. We investigated potential correlations between neutrophil parameters and clinical markers of disease progression.

\section{Methods}

\section{Study design}

This study enrolled 41 patients with acute IS hospitalized in the stroke unit of Saint-Antoine Hospital, Pitié-Salpêtrière Hospital (both Paris, France), and Foch Hospital (Suresnes, France) during the acute phase of brain ischemia. Patients were included if they had had a stroke less than 6 hours before admission, regardless of the NIH Stroke Scale (NIHSS) score, were aged $\geq 18$ years, and showed no signs of infection at the admission. All patients were examined by neurologists, admitted to the stroke unit, and treated according to accepted stroke guidelines. ${ }^{14}$ We prospectively collected the following data for each, with a structured questionnaire: vascular risk factors (age, sex, presence of atrial fibrillation, hypertension, diabetes, hypercholesterolemia, previous stroke history, and treatment); stroke severity assessed by the NIHSS at admission (day 0), day 2, and day 7; and time from symptom onset to brain imaging and sampling. The modified Ranking scale (mRs) was used for functional disability/dependence evaluations at 3 months. Standard stroke evaluation included brain imaging (MRI performed for 37 patients and CT performed for 4 patients when MRI was contraindicated), blood sampling (especially neutrophil and lymphocyte counts), electrocardiography for at least 48 hours, transthoracic echocardiography, and vessel imaging by ultrasound, MR angiography, or angioCT. We prospectively collected stroke information including location of ischemia in the brain, presence and location of 
occlusion, and etiology according to the Trial of Org 10172 in Acute Stroke Treatment classification. IS volumes were measured on diffusion sequences acquired on a 3-Tesla MRI instrument at admission (and not measured for patients with contraindications to brain MRI). A semiautomatic segmentation procedure by Oléa Sphere postprocessing software was used for analysis.

The 22 age-matched recruited healthy controls (HCs), previously presented, ${ }^{16}$ with blood biochemical and hematologic values within the normal range were recruited from the Unit of Neurology of Memory and Language, Centre Hospitalier Sainte Anne (Paris, France). HCs were close members of the family of patients followed in the memory clinic. As previously described, ${ }^{16}$ HCs had no memory complaint or cognitive deficit. In addition, we did not include HCs with a history of IS.

Exclusion criteria in the $\mathrm{HC}$ and patient groups included acute or chronic inflammatory conditions and treatment with corticosteroid or nonsteroidal anti-inflammatory drugs, both of which could modulate neutrophil functions.

Whole blood was sampled, kept on ice, and transported immediately to the laboratory for neutrophil analysis. Importantly, all patients were sampled at inclusion (day 0, D0), within the first 6 hours after IS symptom onset; 14 also had samples taken on D2 and D7.

\section{Standard protocol approvals, registrations, and patient consents}

The Ethics Committee of Saint-Antoine Hospital approved the study, and all patients or their proxies provided written informed consent before participating began. The HCs were recruited as part of their participation in the prospective longitudinal IMABio3 study (PHRC-0054-N 2010), which was approved by the Institutional Review Committee of PitiéSalpêtrière Hospital.

\section{Determination of surface molecule expression on resting neutrophils}

Heparinized whole-blood samples $(500 \mu \mathrm{L})$ were kept on ice and stained with allophycocyanine (APC)-anti-human CD62L (BD Biosciences, San Jose, CA) and PE-anti-human CD11b (clone ICRF44, BD Biosciences) antibodies, as previously reported. ${ }^{15}$ The senescent $\mathrm{CXCR} 4^{\text {bright }} / \mathrm{CD} 62 \mathrm{~L}^{\mathrm{dim}}$ neutrophil subset and the immunosuppressive $\mathrm{CD} 16^{\text {bright }} /$ CD62 ${ }^{\text {dim }}$ neutrophil subset were investigated as previously described,. ${ }^{16}$ To investigate the rTEM neutrophil subset, samples kept on ice were incubated for 45 minutes with FITC-anti-human CD81 (BD Biosciences) and PE-antiCD54 (BD Biosciences) antibodies. The red blood cells were then lysed with BD FACS lysing solution and resuspended with Cell Fix (BD Biosciences). ${ }^{16}$

\section{Measurement of neutrophil oxidative burst}

Superoxide anion $\left(\mathrm{O}_{2}{ }^{-}\right)$production by neutrophils was measured with a flow cytometry-based assay derived from the hydroethidine (HE) oxidation technique, as previously described. ${ }^{16}$ After loading with $\mathrm{HE}$ for 15 minutes, whole-blood samples were incubated for 45 minutes at $37^{\circ} \mathrm{C}$ with phosphate buffered saline (PBS) or either of the following Toll-like receptor (TLR) agonists: $\mathrm{Pam}_{3} \mathrm{CSK}_{4}$ or lipopolysaccharide (LPS), or tumor necrosis factor (TNF)- $\alpha$, as previously reported. ${ }^{22}$ Samples were then treated with PBS or $10^{-6} \mathrm{M}$ $\mathrm{N}$-formylmethionyl-leucyl-phenylalanine (fMLP) for 5 minutes. ${ }^{22}$ Erythrocytes were lysed before analysis by flow cytometry.

\section{Measurement of neutrophil apoptosis and necrosis}

Spontaneous neutrophil cell death in whole blood was quantified with annexin $\mathrm{V}$ and the impermeant nuclear dye 7-amino-actinomycin D (7-AAD). Whole-blood samples $(500 \mu \mathrm{L})$ were incubated with APC-anti-CD15 antibody (clone HI98, BD Biosciences), FITC-annexin V, and 7-AAD (BD Biosciences), as previously described, and analyzed by flow cytometry. ${ }^{16}$

\section{Flow cytometry analysis}

Cells were analyzed with a Gallios flow cytometer and the data with Kaluza software (Beckman Coulter). To determine neutrophil expression of surface molecules and ROS production, forward and side scatter were used to identify the granulocyte population and to gate out other cells and debris. The purity of the gated cells was assessed using FITC- or PEconjugated CD3, CD45, CD14, and CD15 antibodies. Ten thousand events were analyzed per sample, and the fluorescence pulses were amplified by 4 -decade logarithmic amplifiers. In all cases, unstained cells were run, and the photomultiplier settings were adjusted so that the unstained cell population appeared in the lower left-hand corner of the fluorescence display. In the multicolor analysis, single-cell controls were used to optimize signal compensation. All the results were obtained with the use of a constant photomultiplier gain value. To measure cell death in whole blood by flow cytometry, neutrophils were identified as $\mathrm{CD} 15^{\text {high }}$ cells, and $2 \times 10^{5}$ events were analyzed per sample.

\section{Measurement of intravascular NETosis}

NETs are networks of DNA, histone, and proteolytic enzymes produced by activated neutrophils, including myeloperoxidase (MPO), citrullinated histone 3, and elastase. Serum NET levels were quantified by detecting MPO-DNA complexes in serum samples. To prepare the DNA standard, NETs from a healthy subject were produced and isolated, and DNA was quantified in NET samples with PicoGreen. NETassociated MPO-DNA complexes were then quantified, also as previously described. ${ }^{17}$

\section{Measurement of soluble pro- and anti-inflammatory mediators}

Whole-blood samples were centrifuged for 15 minutes at $1,000 \mathrm{~g}$ within 30 minutes of collection. Soluble chemokine (IL8 ) and cytokines (IL-1 $\beta, \mathrm{IL}-6, \mathrm{TNF}-\alpha$, G-CSF, IL-18, IL-22, 
IL-10, and transforming growth factor- $\beta$ ) were detected with Luminex assays (Luminex Performance Assays, R\&D Systems). High-mobility group box-1 (HMGB1) and elastase were detected from serum by ELISA.

\section{Statistics}

Statistical analyses were performed with Rstudio 1.0.143 software. All tests were 2 tailed, with a significance level of $\alpha=$ 0.05 . When a parametric test was used, normality of distribution was tested with the Shapiro-Wilk test. Differences between groups were assessed with the $\chi^{2}$ test or analysis of variance (ANOVA), followed by the Tukey post hoc test, as appropriate. ANOVA, adjusted for age, was used to compare neutrophil markers between the IS groups and controls (with age as a covariate). Bonferroni correction was used for multiple comparisons. Linear partial correlation analysis, with adjustment for covariates, identified correlations.

\section{Data availability statement}

Data not provided will be available upon request.

\section{Results}

\section{Participants' characteristics}

The table summarizes the clinical characteristics of the 41 patients with IS and $22 \mathrm{HCs}$. Patients were included in 2 steps: the results from the first set of 27 patients led us to include a second set of 14 patients for complementary analyses. The 2 patient cohorts did not differ significantly for baseline characteristics, including risk factors. Patients with IS and HCs did not differ significantly for age or sex, but risk factors were significantly more frequent in the IS than the HCs. The proportion of etiologies for cerebral infarction observed in patients appears similar to recent data. ${ }^{18} \mathrm{We}$ observed 4 brainstem strokes (9.8\%), 13 cortical strokes (31.7\%), 11 subcortical strokes (26.8\%), and 13 cortical and subcortical strokes $(31.7 \%)$. Seven patients had hemorrhagic transformation. Five patients died within 7 days of onset. As expected, ${ }^{19}$ patients with IS had higher polymorphonuclear neutrophil (PMN) counts than HCs and higher PMN/ lymphocyte ratios.

\section{Highly activated circulating neutrophils in patients with acute stroke}

We analyzed neutrophil activation in the 27 patients with IS included in the first set of patients and in the $22 \mathrm{HCs}$. Neutrophil activation is associated with the modulation of surface molecules, i.e., decreased L-selectin (CD62L) and increased $\beta 2$-integrins (i.e., CD11b/CD18), through either stimulusinduced shedding or translocation from intracellular granules. As figure 1, A and B show, resting neutrophils from patients on D0 expressed less CD62L and more CD11b than HCs. Because neutrophil degranulation can also result in rapid release of neutrophil serine proteases from azurophilic granules into the extracellular medium and circulation, we measured the level of circulating neutrophil elastase. Consistently with previous data, ${ }^{20}$ serum neutrophil elastase levels were significantly higher in patients with IS than HCs (figure 1C).

Measuring ROS production by neutrophils, we observed it was higher in unstimulated neutrophils from whole blood (PBS-incubated sample) from patients than from HCs (figure 1D). We then assessed whether the higher constitutive ROS production by patient neutrophils was associated with higher production capacity in response to various activating stimuli. We have previously reported that neutrophils in whole blood produce minimal ROS in response to a single stimulus. ${ }^{16} \mathrm{We}$ therefore studied neutrophil oxidative bursts in response to the bacterial peptide fMLP after priming with TNF- $\alpha$ or various TLR agonists. ROS production by nonprimed neutrophils (sample preincubated with PBS and stimulated with fMLP) was significantly higher in patients than in HCs (figure $1 \mathrm{E})$, and under the different priming conditions, the significantly higher ROS production in patients with IS (figure 1E) demonstrates their neutrophil hyperreactivity.

Among the patients included in our study, 14 were also sampled on D2 and D7. Except for CD62L expression, which returned to normal levels on those days, no markers of neutrophil activation were modulated from D0 during the course of acute IS (figure 1, F-J). No significant differences among any of the neutrophil activation markers were observed between patients with or without subsequent stroke-associated infections or patients with or without IS risk factors (such as hypertension, diabetes, and hyperlipidemia) (data not shown).

These results reflect basal hyperactivation of patients' circulating neutrophils during acute IS, associated with their hyperreactivity to various stimuli by ROS production.

\section{Deregulation of neutrophil death in patients with acute stroke}

Neutrophils constitutively undergo apoptosis, and this process is critical for the resolution of inflammation. Neutrophil death by necrosis, inversely, induces the release of cytotoxic mediators that can damage adjacent healthy tissue. Measuring the percentage of neutrophils undergoing spontaneous apoptosis and necrosis showed that the percentage of apoptotic neutrophils at D0 was significantly lower in patients with IS and tended to return to normal values during the course of IS (figure 2, A and B). The percentage of necrotic neutrophils, however, was rising at D0, and the increase became significant from D2 (figure 2, C and D). This deregulation of the balance between apoptosis and necrosis, combined with basal neutrophil hyperactivation, may play a role in establishing inflammatory processes in these patients.

Neutrophils can undergo NETosis, an alternative form of programmed cell death associated with the release of decondensed chromatin lined with granular components that creates fibrous structures serving as NETs. These NETs can entangle pathogens but also contribute to both venous and arterial thrombosis. ${ }^{21}$ Patients with IS had lower levels of 
Table Characteristics of patients with ischemic stroke and HCs participating in the study

\begin{tabular}{|c|c|c|c|c|c|c|}
\hline & HC $(n=22)$ & $\begin{array}{l}\text { Patient set } 1 \\
(n=27)\end{array}$ & $\begin{array}{l}\text { Patient set } 2 \\
(n=14)\end{array}$ & $\begin{array}{l}p \text { Value, set } 1 \\
\text { vs HC }\end{array}$ & $\begin{array}{l}p \text { Value, set } 2 \\
\text { vs HC }\end{array}$ & $\begin{array}{l}p \text { Value, set } 1 \\
\text { vs set } 2\end{array}$ \\
\hline Sex (male), (n) [\%] & $6[35.3]$ & $14[51.8]$ & $8[57.14]$ & 0.36 & 0.29 & 0.99 \\
\hline Age, yr, (mean) [min-max] & $68.1[22-89]$ & 72.6 [18-96] & $71.1[40-92]$ & 0.20 & 0.54 & 0.59 \\
\hline \multicolumn{7}{|l|}{ History of: (n) [\%] } \\
\hline Diabetes & $1[4.54]$ & $6[22.2]$ & $1[5.89]$ & 0.11 & 0.99 & 0.69 \\
\hline High blood pressure & $2[11.8]$ & $21[77.8]$ & $10[71.4]$ & 0.001 & 0.001 & 0.71 \\
\hline Dyslipidemia & $4[23.5]$ & 14 [51.9] & $2[14.3]$ & 0.02 & 0.99 & 0.69 \\
\hline Myocardial infarction & 0 & $9[33.3]$ & $4[28.6]$ & 0.002 & 0.02 & 0.99 \\
\hline Atrial fibrillation & 0 & $8[29.6]$ & $4[28.6]$ & 0.006 & 0.02 & 0.99 \\
\hline Stroke & 0 & $6[22.2]$ & $1[7.14]$ & 0.026 & 0.39 & 0.39 \\
\hline NIHSS DO (mean) [min-max] & - & $9.15[0-21]$ & $8.64[1-23]$ & - & - & 0.64 \\
\hline \multicolumn{7}{|l|}{ Etiologies/TOAST (n) [\%] } \\
\hline Thromboembolism & - & $6[22.2]$ & $2[14.3]$ & - & - & 0.69 \\
\hline Cardioembolism & - & $11[40.7]$ & $4[28.6]$ & - & - & 0.51 \\
\hline Lacunar stroke & - & $1[3.7]$ & $2[14.3]$ & - & - & 0.54 \\
\hline Other specific cause & - & $3[7.4]$ & $3[21.4]$ & - & - & 0.64 \\
\hline Undetermined cause & - & $6[22.2]$ & $3[21.4]$ & - & - & 0.99 \\
\hline Occlusion at inclusion $(\mathrm{n})[\%]$ & - & 16 [59.3] & $6[42.8]$ & - & - & 0.51 \\
\hline $\begin{array}{l}\text { Volume of cerebral infarct (mL) (mean) } \\
\text { [min-max] }\end{array}$ & - & $\begin{array}{l}23.92 \\
{[0.04-240.8]}\end{array}$ & $16.17[0-63.8]$ & - & - & 0.99 \\
\hline Treatment at acute stage $(n)[\%]$ & - & & & & & \\
\hline IV Thrombolysis & - & $17[62.9]$ & $5[35.7]$ & - & - & 0.115 \\
\hline Endovascular treatment & - & $9[33.3]$ & $4[28.6]$ & - & - & 0.99 \\
\hline $\begin{array}{l}\text { Time from symptom onset to blood sample } \\
\text { (min) (mean) [min-max] }\end{array}$ & - & $129[45-240]$ & $186[95-340]$ & - & - & 0.09 \\
\hline Previous treatment by statins & $2[11.7]$ & $9[33.3]$ & $3[21.4]$ & 0.158 & 0.636 & 0.494 \\
\hline $\begin{array}{l}\text { Previous treatment by antithrombotic } \\
\text { agents }\end{array}$ & 0 & $8[29.6]$ & $4[28.6]$ & 0.006 & 0.017 & 0.99 \\
\hline Neutrophil count (G/L) (mean) [min-max] & $\begin{array}{l}3.15 \\
{[2.32-4.80]}\end{array}$ & $\begin{array}{l}6.1 \\
{[2.37-14.40]}\end{array}$ & $\begin{array}{l}7.85 \\
{[2.33-15.3]}\end{array}$ & 0.007 & 0.002 & 0.22 \\
\hline Lymphocyte count (G/L) (mean) [min-max] & $\begin{array}{l}1.68 \\
{[1.06-2.15]}\end{array}$ & $\begin{array}{l}1.48 \\
{[0.53-3.15]}\end{array}$ & $\begin{array}{l}1,52 \\
{[0.57-3.26]}\end{array}$ & 0.43 & 0.55 & 0.91 \\
\hline Ratio PMN/lymphocyte (mean) [min-max] & $\begin{array}{l}2.09 \\
{[1.37-4.36]}\end{array}$ & $\begin{array}{l}5.63 \\
{[1.04-23.34]}\end{array}$ & $\begin{array}{l}7.70 \\
{[1.04-26.84]}\end{array}$ & 0.023 & 0.024 & 0.40 \\
\hline
\end{tabular}

Abbreviations: $\mathrm{HC}=$ healthy control; NIHSS = NIH Stroke Scale; TOAST = Trial of Org 10172 in Acute Stroke Treatment.

Sex, risk factors, and type of treatment were compared with the $\chi^{2}$ test. The Mann-Whitney test was used to compare quantitative variables.

We considered all thrombolyses and all mechanical thrombectomies.

circulating NETosis-derived products in their blood samples at inclusion than HCs (figure 2E). This difference persisted at D2 and D7 (figure 2F).

Patients with or without either infection or IS risk factors did not differ significantly for neutrophil apoptosis, necrosis, or intravascular NETosis (data not shown).

\section{Expansion of the overactive senescent} neutrophil subset in patients with acute stroke The phenotypes and functions of neutrophils change over time from their release into the blood (as fresh neutrophils) until their disappearance from the circulation (as aged neutrophils). Aged or senescent CXCR4 ${ }^{\text {high }}$ neutrophils express lower levels of CD62L and higher levels of $\beta 2$-integrins and produce ROS at a higher rate 
A

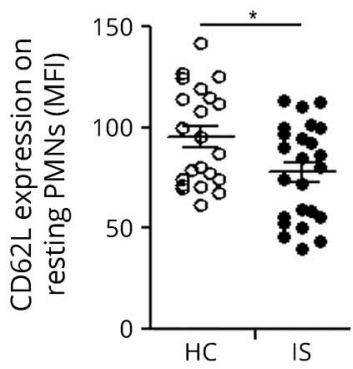

D

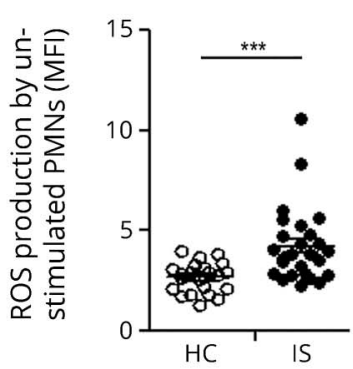

F

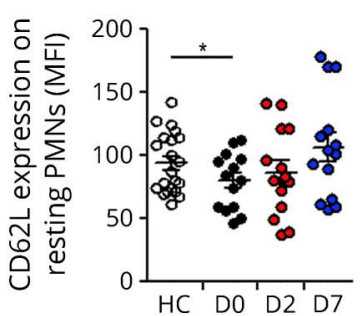

I

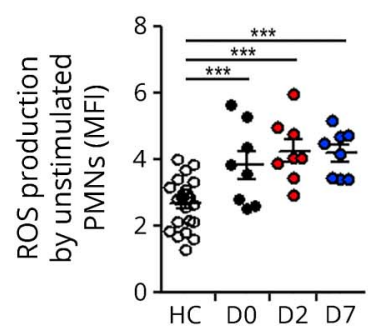

B

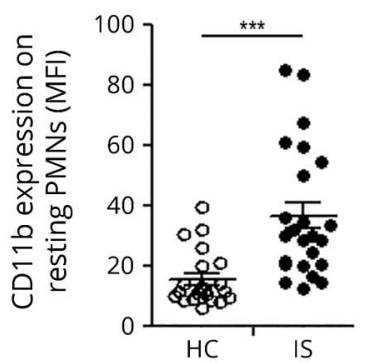

C

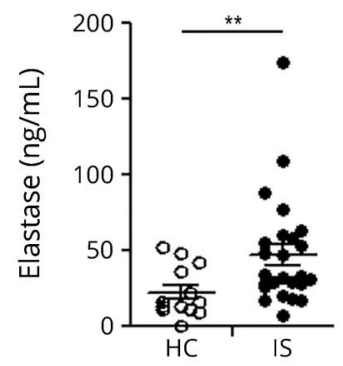

E

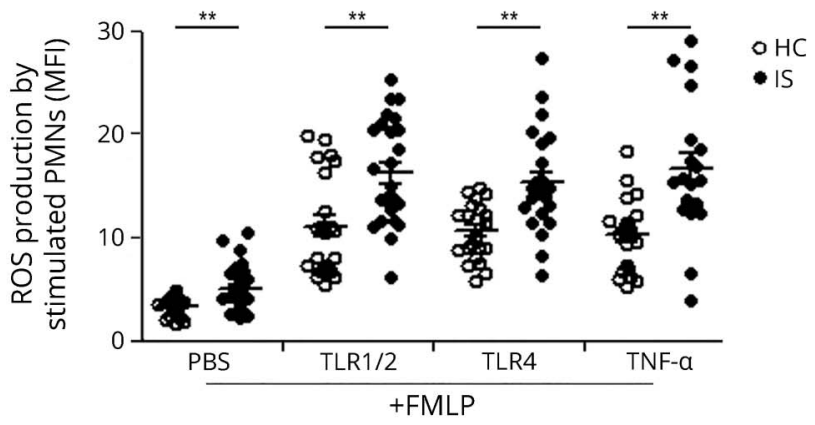

G
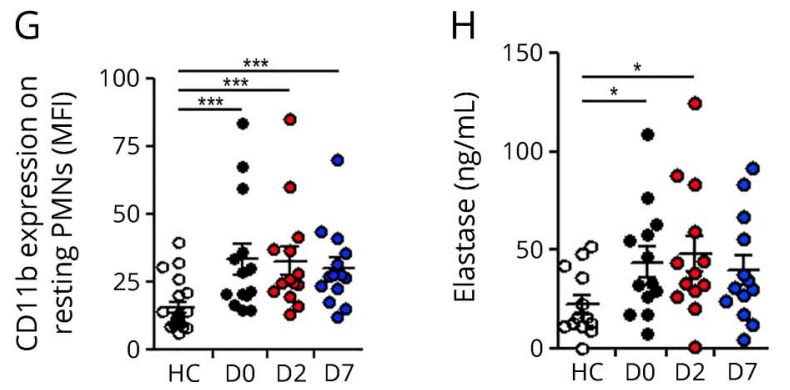

J

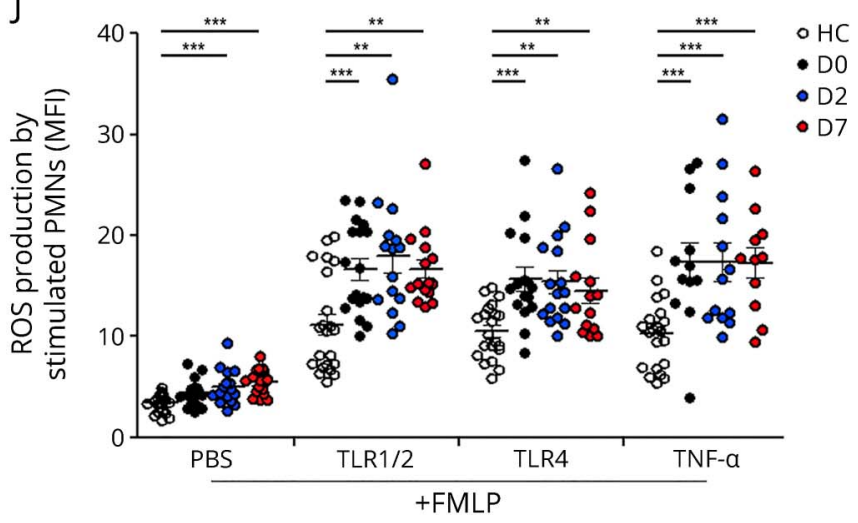

(A-E) Adhesion molecules expression, polymorphonuclear neutrophil (PMN) elastase, and ROS production were studied in patients with IS at inclusion. Expression of CD62L (A) and CD11b (B) at the surface of resting PMNs was studied on whole-blood samples kept on ice and incubeted with anti-CD62L and antiCD11b monoclonal antibodies. Results are expressed as mean fluorescence intensity (MFI). (C) Circulating levels of PMN elastase were quantified by ELISA; results are expressed as ng/mL. ( $D$ and E) ROS production by unstimulated PMNs was studied after treatment of wholeblood samples for 50 minutes with PBS; results are expressed as MFI (D). ROS production by stimulated PMNs was measured after pretreatment of whole-blood samples for 45 minutes with PBS, $\mathrm{Pam}_{3} \mathrm{CSK}_{4}$ (TLR1/2 agonist, $1 \mu \mathrm{g} / \mathrm{mL}$ ), or LPS (TLR4 agonist, $10 \mathrm{ng} /$ $\mathrm{mL})$, or TNF-a (TNF, $5 \mathrm{ng} / \mathrm{mL})$ and incubation for 5 minutes with fMLP $\left(10^{-6} \mathrm{M}\right)$; results are expressed as MFI (E). (F-J) Follow-up of surface expression of CD62L (F), surface expression of CD11b $(G)$, circulating levels of PMN elastase $(H)$, and ROS production by unstimulated (I) and stimulated PMNs (J) during the first week after IS symptoms. All samples came from age-matched HCs, $(n=22)$ and patients with IS at day $0(n=27)$, day 2 $(n=14)$, and day $7(n=14)$. Values are mean \pm SEM. *Significantly different from controls $p<0.05, * \star p<0.01, * \star * p$ $<0.001$, adjusted for age. $\mathrm{DO}=$ day 0 ; $\mathrm{D} 2$ = day $2 ; \mathrm{D} 7=$ day $7 ; \mathrm{fMLP}=\mathrm{N}$-formylmethionyl-leucyl-phenylalanine; $\mathrm{HC}=$ healthy control; IS = ischemic stroke; LPS = lipopolysaccharide; ROS = reactive oxygen species; $S E M=$ standard error of mean; TLR = Toll-like receptor; TNF = tumor necrosis factor. than the total circulating pool. ${ }^{8}$ Conversely, in contexts of acute inflammation ${ }^{9}$ and healthy aging, ${ }^{22}$ an immunosuppressive subset of $\mathrm{CD} 16^{\text {bright }} / \mathrm{CD} 62 \mathrm{~L}^{\mathrm{dim}}$ neutrophils show decreased adhesion properties and less ROS production. Changes in the phenotypic and functional features of patients' neutrophils might thus be related to an imbalance between these 2 neutrophil subsets. The senescent CXCR4 ${ }^{\text {bright }} / \mathrm{CD} 62 \mathrm{~L}^{\mathrm{dim}}$ neutrophil subset expanded in patients with IS at D0 (figure 3, A and C), whereas the percentage of neutrophils in the $\mathrm{CD} 16^{\text {bright }} / \mathrm{CD} 62 \mathrm{~L}^{\mathrm{dim}}$ immunosuppressive subset did not differ between patients with IS and HCs (figure 3, B and D). Accordingly, patients with IS had a higher ratio of senescent to immunosuppressive subsets than HCs (figure 3, E). Similar results were observed in patients with IS at D2 and D7 (figure 3, F-H). Moreover, PMN subsets did not differ significantly between patients with or without infection or IS risk factors (data not shown). These results reflect the expansion of the senescent subset in patients with IS may lead to greater PMN oxidative burst. 
Figure 2 Deregulation of neutrophil death in patients with ischemic stroke
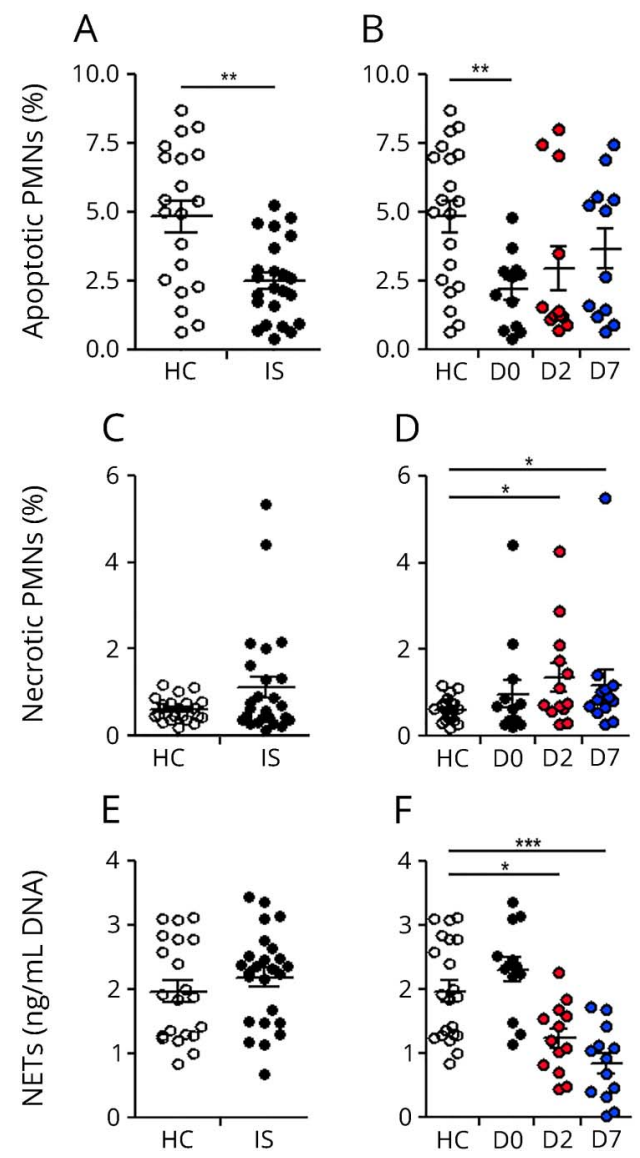

F

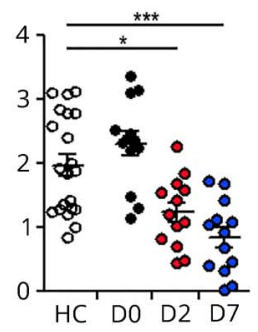

(A-D) Polymorphonuclear neutrophil (PMN) death was measured immediately after sampling by staining with annexin $\mathrm{V}$ and 7-AAD. Results are expressed as the percentages of apoptotic ( $A$ and $B$ ) and necrotic ( $C$ and $D$ ) PMNs. ( $A$ and $C$ ) Values of PMN death in patients with IS at inclusion. (B and D) Follow-up of PMN death during the first week after IS symptoms. (E and F) Circulating NETs were quantified by MPO-DNA complex ELISA; results are expressed as ng/ $\mu \mathrm{L}$ DNA. Values of circulating NETs in patients with IS at inclusion (E). Follow-up of intravascular NETosis during the first week after IS symptoms (F). All samples came from age-matched healthy controls ( $\mathrm{HCs}, \mathrm{n}$ $=22)$ and patients with IS at day $0(n=27)$, day $2(n=14)$, and day $7(n=14)$. Values are mean \pm SEM. *Significantly different from controls $p<0.05$, ** $p<$ $0.01, * \star \star p<0.001$, adjusted for age. AAD = amino-actinomycin D; D0 = day 0; $\mathrm{D} 2=$ day $2 ; \mathrm{D} 7=$ day $7 ; \mathrm{HC}=$ healthy control; IS = ischemic stroke; $\mathrm{MPO}=$ myeloperoxidase; NET = neutrophil extracellular trap; SEM = standard error of mean.

Increased levels of soluble JAM-C were associated with an increase in the rTEM neutrophil subset in patients with

\section{acute stroke}

JAM-C is an adhesion molecule expressed by endothelial cells that plays a role in tight junction formation, leukocyte adhesion, and transendothelial migration. Elastase-induced proteolytic cleavage of endothelial JAM-C leading to soluble JAM-C (sJAM-C) was recently reported to be instrumental in promoting neutrophil rTEM in vivo. ${ }^{23}$ Measuring the circulating levels of JAM-C in our first cohort of 27 patients with IS showed a higher level at D0 than in $\mathrm{HCs}$ (figure 4A); values returned to normal at D2 (figure 4B). As expected, ${ }^{23}$ increased sJAM-C levels were associated with an increased circulating level of neutrophil elastase (figure 4C).

We thus performed a complementary study in a second cohort of 14 patients with IS to investigate the percentage of circulating rTEM neutrophils and its association with the sJAM-C level. The clinical characteristics of this second cohort did not differ from that of the main cohort (table), nor did their sJAM-C level on D0 $(421 \pm 20 \mathrm{pg} / \mathrm{mL}$ and $463 \pm 49$ $\mathrm{pg} / \mathrm{mL}$ for the patient set 1 and set 2 , respectively, $p=0.77$ ), which was similarly significantly higher than in HCs. As previously reported, ${ }^{23}$ a small population of neutrophils with an rTEM phenotype $\left(\mathrm{CD} 54^{\text {high }}, \mathrm{CXCR} 1^{\text {low }}\right)$ was consistently found in HCs, equivalent to $1 \%$ of all circulating neutrophils. In patients with IS, we could also identify a distinct population of cells with an rTEM phenotype; its percentage was significantly higher in patients than in HCs (figure 4, D and E), and the increase of this percentage was associated with increased levels of sJAM-C (figure 4F). Taken together, these results demonstrate the expansion of the rTEM subset in IS migration.

\section{Neutrophil abnormalities are associated with the severity of acute IS}

We then investigated whether the neutrophil abnormalities observed in patients with IS might be associated with stroke severity. Patients were dichotomized according to the NIHSS score at inclusion into minor-to-moderate (NHISS score $\leq 12$ ) and severe stroke (NHISS score $>12$ ) categories. Basal neutrophil activation at baseline was greater in severe than in minor-to-moderate strokes, as shown by patients' lower CD62L expression (figure 5A), higher circulating neutrophil elastase levels (figure 5C), and higher neutrophil production of ROS (figure 5D), although CD11b expression did not differ significantly between the groups (figure 5B). These phenotypic and functional properties of neutrophils from the severe stroke group were associated with a higher percentage of neutrophils in the senescent hyperactive subset (figure 5E), but not in the immunosuppressive subset (figure 5F). Finally, the sJAM-C level was higher in the severe stroke group (figure $5 \mathrm{G}$ ). In contrast, the percentage of apoptotic (figure $5 \mathrm{H}$ ) and necrotic neutrophils (data not shown) and the intravascular NETosis level (data not shown) did not differ between the patient groups. Higher NIHSS scores at D0 were associated with decreased expression of CD62L (figure 5I), increased ROS production by both unstimulated (figure 5J) and LPSprimed neutrophils (figure 5K), and an increase in the percentage of the senescent hyperactive subset (figure 5L), the level of sJAM-C (figure 5M), and the percentage of rTEM neutrophils (figure $5 \mathrm{~N}$ ). Increased NIHSS score at D2 was also associated with increased ROS production by LPS-primed neutrophils (data not shown). The correlations were adjusted for age. We found no correlation between neutrophil parameters and the infarct volume at inclusion and the presence of macroangiopathy, the occurrence of hemorrhagic transformation, the use of antithrombotic treatment, or the use of thrombolysis or endovascular treatment (data not shown). 
A

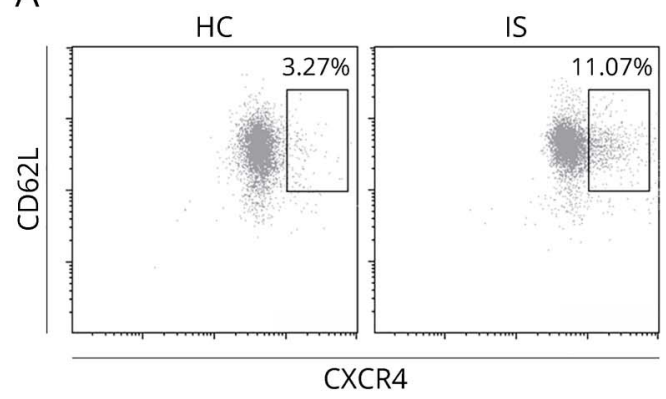

B

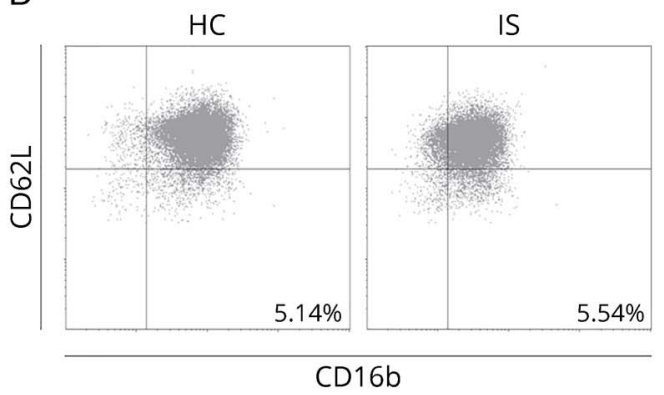

E
C

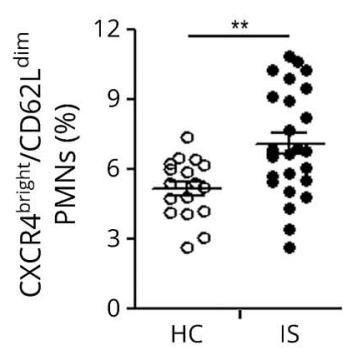

F

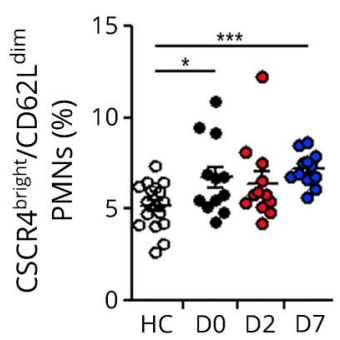

D

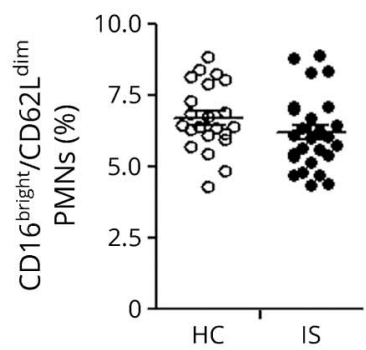

G

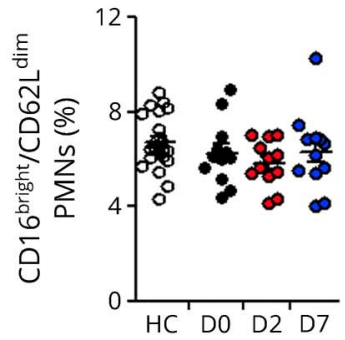

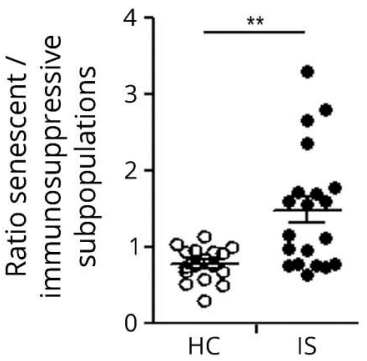

$\mathrm{H}$

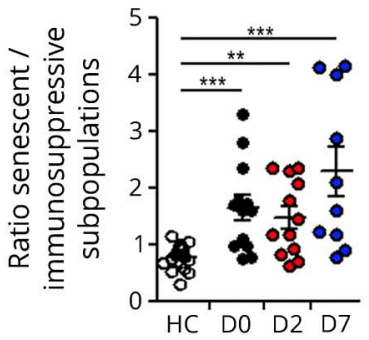

(A-E) Analysis of senescent and immunosuppressive polymorphonuclear neutrophil (PMN) subsets in patients with IS at inclusion. Whole-blood samples were incubated for 45 minutes at $4^{\circ} \mathrm{C}$ with Pe-Cy7-anti-human CXCR4, PE-anti-human CD11b, and APC-antihuman CD62L (A and B) or with FITC-anti-human CD16, PE-anti-human CD11c, Pe-Cy7-antihuman CD11b, and APC-anti-human CD62L (C and D) antibodies. (A) Representative dot plots of the PMN phenotype according to CXCR4 and CD62L expression in a HC (left) and a patient with IS (right). (B) Representative dot plots of the PMN phenotype according to CD16 and CD62L expression in an HC (left) and a patient with IS (right). (C) Percentages of the CXCR4 ${ }^{\text {bright }} / C D 62 L^{\text {dim }}$ senescent PMN subset. (D) Percentages of the CD16 bright/CD62L dim immunosuppressive PMN subset. (E) Ratio between the senescent and the immunosuppressive PMN subsets. (F-H) Follow-up of senescent PMN subset (F), immunosuppressive PMN subset $(G)$, and ratio between the senescent and the immunosuppressive PMN subsets (H) during the first week after IS symptoms. All samples came from age-matched HCs, $(n=22)$ and patients with IS at day $0(n=27)$, day $2(n=14)$, and day $7(n=14)$. Values are mean \pm SEM. *Significantly different from controls $p<0.05,{ }^{* \star} p<0.01$, ${ }^{* \star} p<0.001$, adjusted for age. D0 = day $0 ; \mathrm{D} 2=$ day $2 ; \mathrm{D} 7=$ day $7 ; \mathrm{HC}=$ healthy control; IS $=$ ischemic stroke; SEM = standard error of mean.

Considered together, these results strongly suggest that the impaired homeostasis of circulating neutrophils associated with neutrophil hyperactivation is correlated with the clinical severity of acute stroke disease progression.

\section{Proinflammatory environment in patients with acute stroke}

Ischemic cerebral tissue rapidly induced the release of cytokines, chemokines, and danger-associated molecular patterns (DAMPs), including HMGB1. ${ }^{24}$ Because cytokines $^{25}$ and $\mathrm{HMGB}^{26}$ strongly regulate neutrophil activity, we investigated patients' peripheral proinflammatory environment. Levels of classic proinflammatory (IL-1 $\beta$, IL-6, IL-8, and TNF $\alpha$ ) and anti-inflammatory (IL-10 and transforming growth factor- $\beta$ ) cytokines were similar in the IS patient and HC groups (figure 6A). Serum levels of G-CSF and IL-22, a bifunctional cytokine with both proinflammatory and protective functions, were significantly lower in the IS than the HC group. Inversely, serum levels of the proinflammatory cytokine IL-18 were higher in the patients with IS (figure 6A), as were circulating HMGB1 levels (figure 6, B and $\mathrm{C}$ ), which were associated with the clinical NIHSS score on D0 (figure 6D). Taken together, these results demonstrate higher circulating levels of the proinflammatory cytokine IL-18 and of the DAMP HMGB-1 in patients with IS.

We then investigated correlations between neutrophil parameters and proinflammatory mediator levels. Neutrophil markers were not correlated with cytokine levels. In 

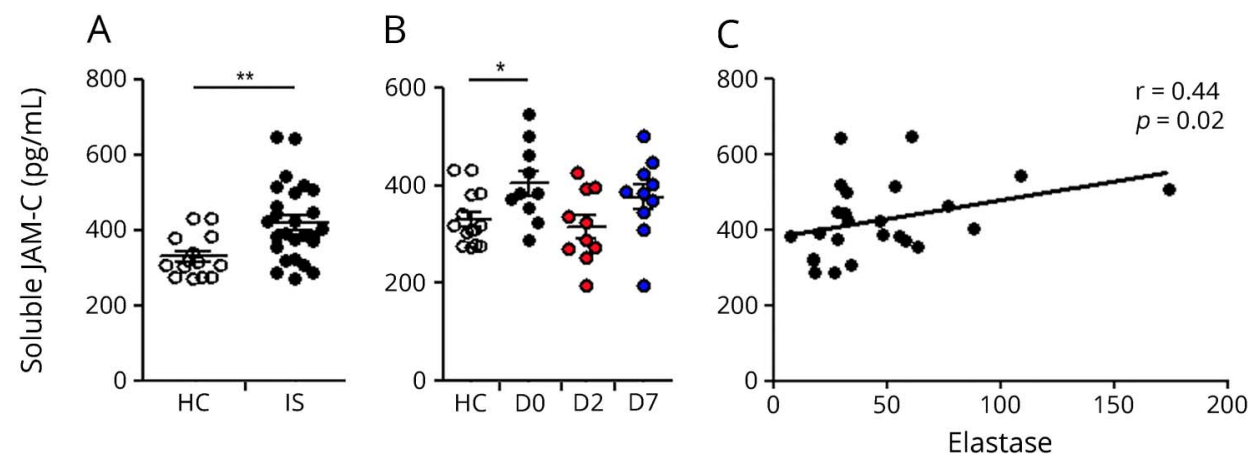

D
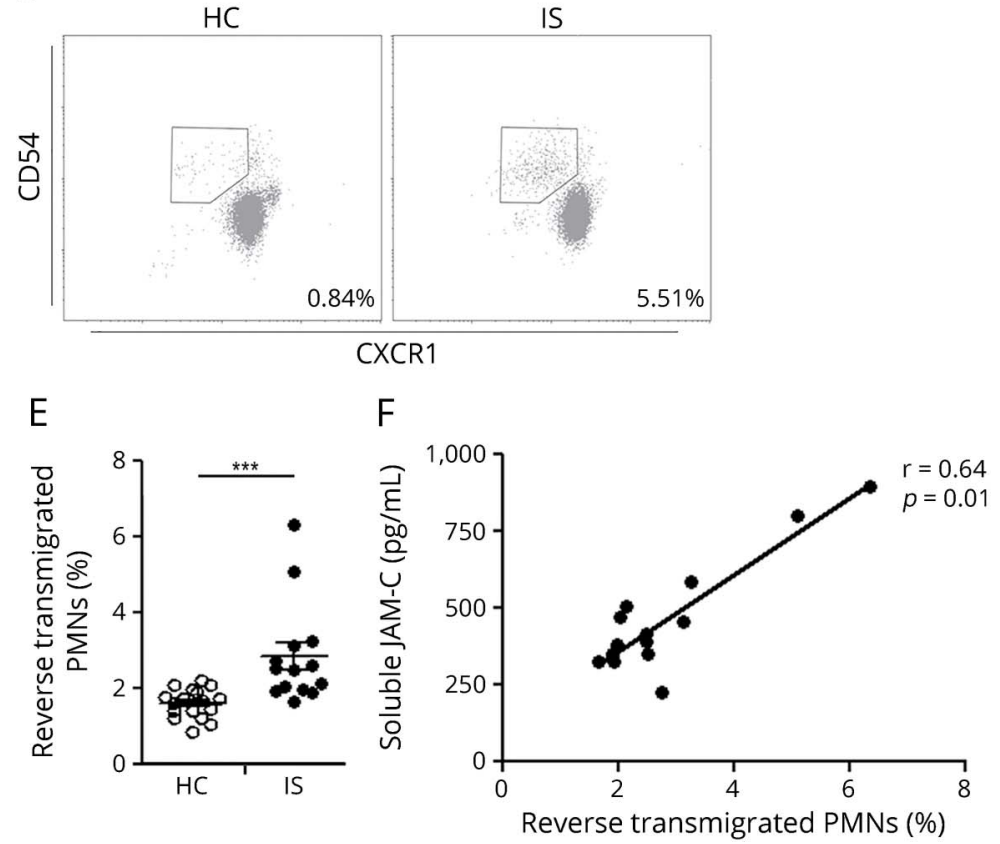

(A and B) SJAM-C was quantified by ELISA; results are expressed as $\mathrm{pg} / \mathrm{mL}$. (A) Values of SJAM-C in age-matched HCs, $(n=22)$ and patients with IS at inclusion ( $n=27$ ) (patients included in set 1). (B) Follow-up of SJAM-C levels during the first week after IS symptoms in 10 patients. (C) Correlation between the levels of SJAM-C and of circulating PMN elastase. ( $D$ and $E$ ) Quantification of rTEM PMNs in patients with IS included in set 2. Whole-blood samples were incubated for 45 minutes at $4^{\circ} \mathrm{C}$ with FITCanti-human CD181 and PEanti-human CD54 antibodies. (D) Representative dot plots of the PMN phenotype according to CXCR4 and CD62L expression in an HC (left) and a patient with IS (right). (E) Percentages of the rTEM PMN subset (CD54 $4^{\text {nigh }}$, CXCR $\left.1^{\text {low }}\right)$ in age-matched HCs $(n=17)$ and patients with IS at inclusion $(n=14)$. (F) Correlation between the percentage of rTEM PMN subset and the level of SJAM-C in patients with IS included in the set 2. *Significantly different from controls $p<0.05, * \star p<0.01, * \star \star x p<$ 0.001 , adjusted for age. $\mathrm{D} 0=$ day $0 ; \mathrm{D} 2=$ day $2 ; \mathrm{D} 7=$ day $7 ; \mathrm{HC}=$ healthy control; IS = ischemic stroke; PMN = polymorphonuclear neutrophil; rTEM = reverse transendothelial migration; SJAM-C = soluble JAM-C. contrast, the increased serum HMGB1 level was associated with decreased $\mathrm{CD} 62 \mathrm{~L}$ expression on resting neutrophils (figure 6E), increased $\mathrm{CD} 11 \mathrm{~b}$ expression (figure 6F), and increased ROS production by primed neutrophils (figure $6 \mathrm{G})$. These findings suggest that HMGB1 might preactivate neutrophils in vivo.

\section{Discussion}

In the present study, phenotypes and functions of peripheral neutrophils from patients less than 6 hours after IS have been extensively investigated. Our results demonstrated continuous basal hyperactivation of circulating neutrophils during acute IS, characterized by lower L-selectin expression and higher CD11b expression at the cell surface, increased ROS production by neutrophils, and greater circulating levels of neutrophil elastase. The release of this soluble cytotoxic molecule might induce vascular damage that aggravates ischemia or diffuses into the brain parenchyma and causes tissue injury. Neutrophil hyperactivation was associated with deregulation of the equilibrium between apoptotic and necrotic PMNs, which may be a supplemental factor promoting acute ischemic inflammation. Furthermore, the lack of difference in neutrophil changes according to the presence or absence of risk factors for stroke, such as type 2 diabetes and hypertension, suggests that these abnormalities might be directly linked to IS.

Activated neutrophils may contribute to vascular inflammation during IS through neutrophil-induced BBB impairment. The BBB forms a protective barrier around the brain, with the essential function of maintaining brain homeostasis. During ischemia and subsequent reperfusion, its integrity is disrupted, which results in the leakage of blood-borne proteins into brain tissue. Loss of $\mathrm{BBB}$ integrity correlates with poorer long-term outcomes in patients with IS. ${ }^{27}$ ROS, including those produced extracellularly by neutrophils, ${ }^{28}$ have been reported to induce BBB breakdown. ${ }^{29}$ In particular, ROS activate matrix 
A

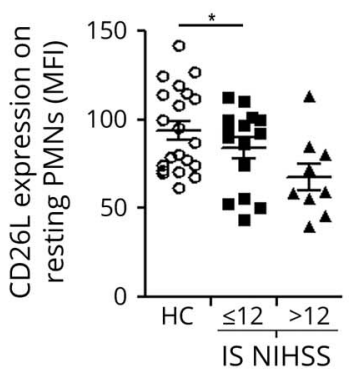

E

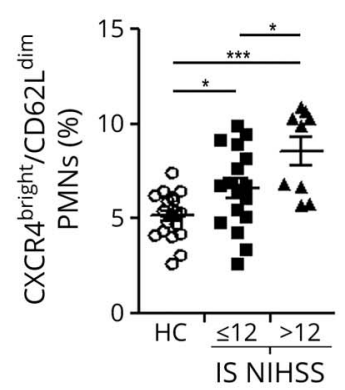

I

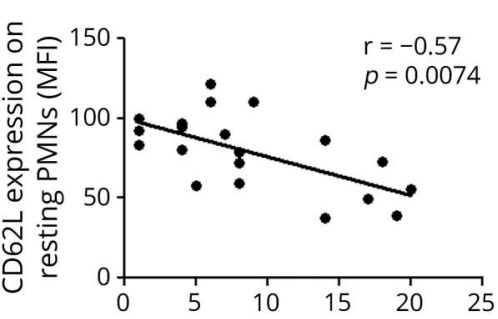

L

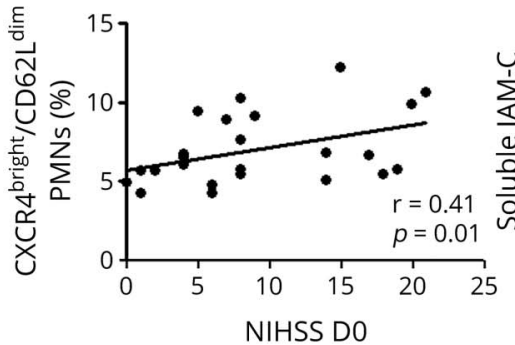

$M$
C

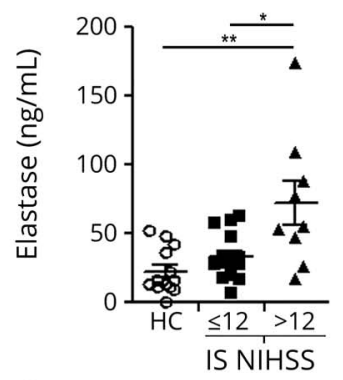

G

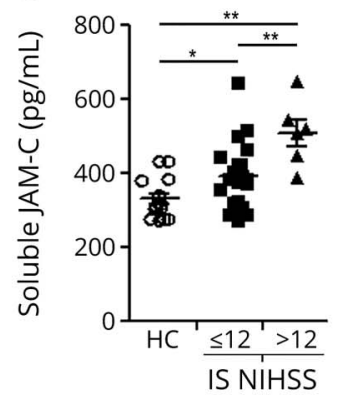

D

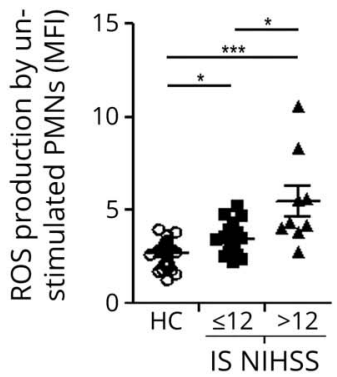

$\mathrm{H}$

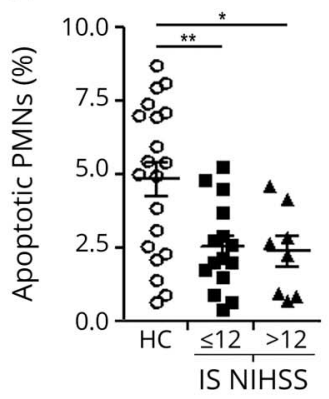

$\mathrm{K}$
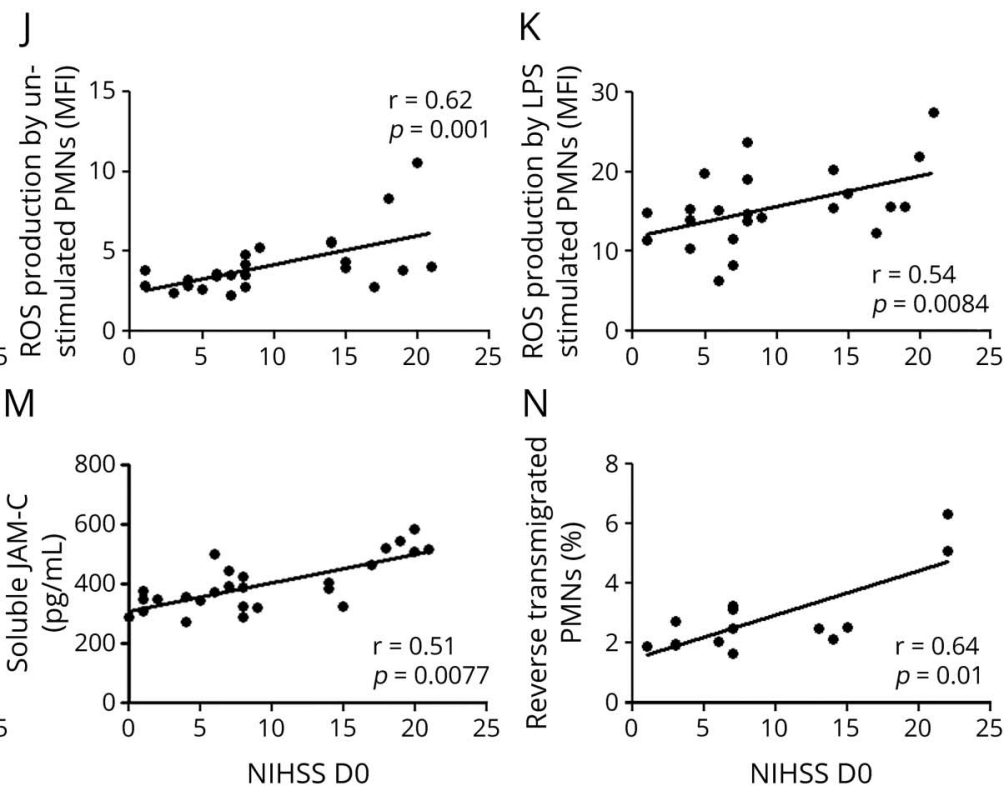

(A and B) Expression of CD62L (A) and CD11b (B) at the surface of resting polymorphonuclear neutrophils (PMNs). Results are expressed as MFI. (C) Circulating levels of neutrophil elastase were quantified by ELISA. (D) ROS production by unstimulated PMNs; results are expressed in MFI. (E) Percentages of the

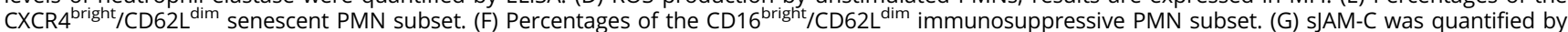
ELISA; results are expressed as pg/mL $(\mathrm{H})$ PMN apoptosis was measured immediately after sampling by staining with annexin $V$ and 7-AAD. Results are expressed as percentages of apoptotic PMNs. All measurements came from patients with minor-to-moderate stroke (IS NHISS $\leq 12, \mathrm{n}=17$ ) and patients with severe stroke (IS NHISS $>12, \mathrm{n}=10$ ) at the inclusion. Values are mean \pm SEM. Statistical significance as determined by the nonparametric Mann-Whitney test is indicated. *Significantly different $p<0.05, * \star p<0.01, * \star \star p<0.001$. Correlation between the NIHSS score at inclusion and CD62L expression at the PMN surface (I), ROS production by unstimulated PMNs (J), ROS production by LPS-stimulated PMNs (K), percentage of the CXCR4 $4^{\text {bright } / C D 62 L^{\text {dim }}}$ senescent PMN subset (L), sJAM-C level (M), and percentage of rTEM PMNs (N). AAD = amino-actinomycin D; D0 = day 0; IS = ischemic stroke; LPS = lipopolysaccharide; MFi = mean fluorescence intensity; NIHSS = NIH Stroke Scale; PMN = polymorphonuclear neutrophil; ROS = reactive oxygen species; SEM = standard error of mean; sJAM-C = soluble JAM-C.

metalloproteinase (MMP) activation, which in turn plays a key role in $\mathrm{BBB}$ breakdown by disrupting the basement membrane of the neurovascular unit. ${ }^{30}$ Among the various MMPs, MMP-9 (gelatinase B) is thought to be central to BBB impairment. Neutrophils are the major source of MMP-9 after IS, and neutrophil-derived MMP-9 has been shown to be important in postischemic $\mathrm{BBB}$ disruption, leukocyte infiltration, and brain damage. ${ }^{29} \mathrm{BBB}$ disruption triggers inflammatory response and infiltration of immune cells including neutrophils, which are in turn associated with increased neuronal cell death through the 


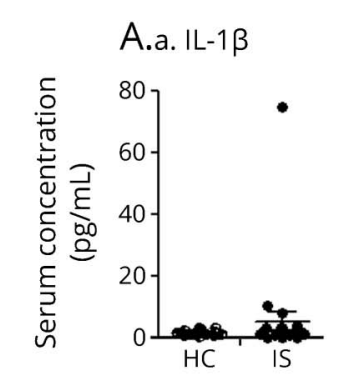

A.f. G-CSF

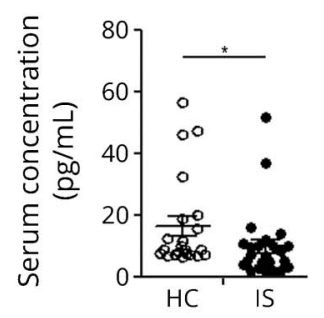

B. HMGB1

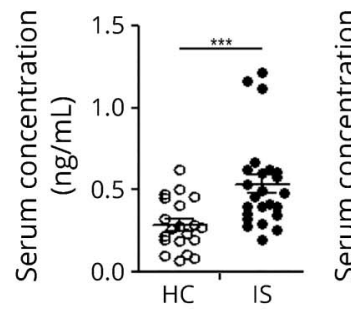

E

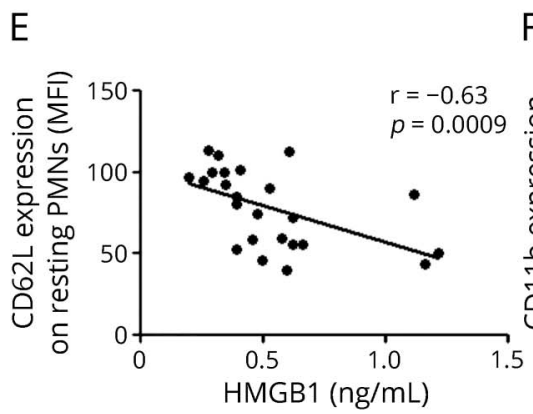

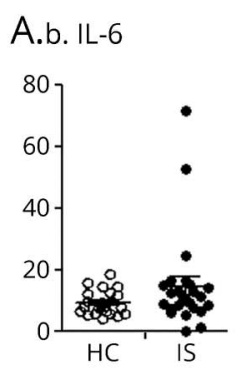

A.g. IL-22

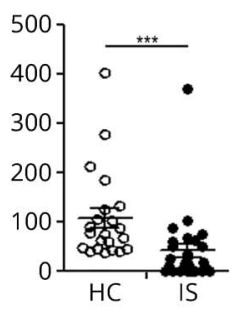

C. HMGB1

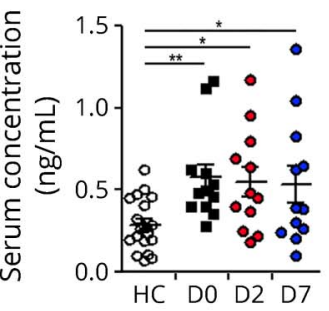

F

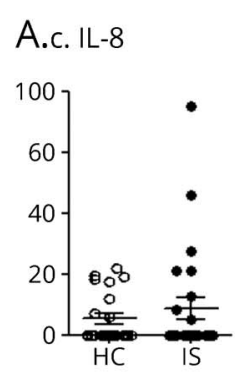

A.h. IL-10

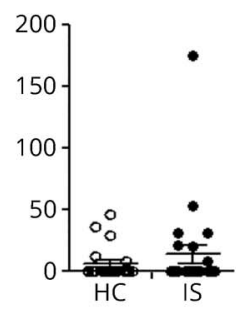

A.d. TNF-a

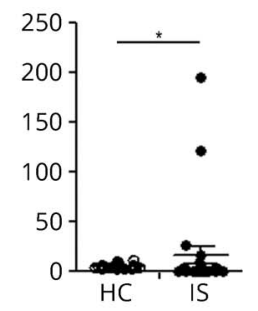

A.i. TGF- $\beta$

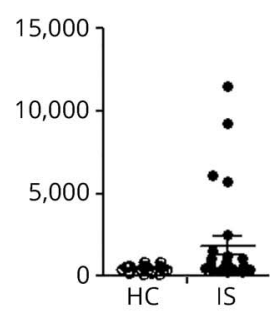

A.e. IL-18

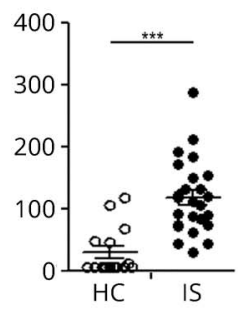

D

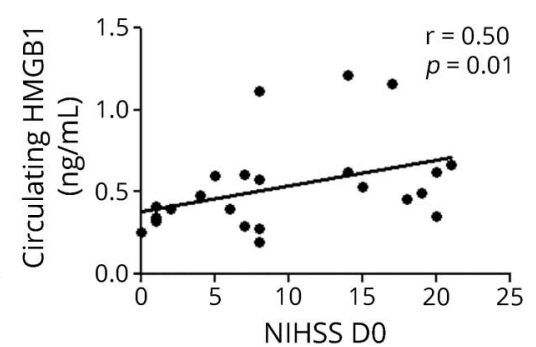

G

A) Circulating levels of pro- and anti-inflammatory cytokines were measured with Luminex assays in samples from controls ( $\mathrm{HCS}$, $\mathrm{n}=22$ ) and patients with IS at day $0(n=27)$. (B and C) Circulating levels of HMGB1 were measured in serum by ELISA. (B) Comparative analysis of circulating levels of HMGB1 between age-matched HCS $(n=22)$ and patients with IS (IS) at day $0(n=27)$. (C) Follow-up of circulating levels of HMGB1 in patients with IS at day 0,2 , and 7. Values are mean \pm SEM. *Significantly different from controls $p<0.05, * \star p<0.01, * \star \star p<0.001$, adjusted for age. Correlation between circulating levels of HMGB1 at day 0 and the NIHSS score at day 0 (D). (E-G) Correlation between circulating levels of HMGB1 at day 0 and PMN activation markers at day 0 : PMN surface expression of CD62L (E) and of CD11b (F) and ROS production by LPS-stimulated PMNs (G). D0 = day 0; D2 = day 2; D7 = day 7; HC = healthy control; HMGB = high-mobility group box; IS = ischemic stroke; LPS = lipopolysaccharide; NIHSS = NIH Stroke Scale; PMN = polymorphonuclear neutrophil; ROS = reactive oxygen species; SEM = standard error of mean.

release of neurotoxic substances and secondary injuries. As senescent neutrophils in the circulation have been reported to rapidly migrate to the inflammatory site, ${ }^{31}$ we speculate that the expansion of the senescent neutrophil subset observed in patients with IS might result in enhanced migration of these hyperactive cells in the brain. Both the percentage of senescent neutrophils and the parameters of neutrophil hyperactivation were correlated with disease severity measured by the NIHSS score. We did not, however, find any correlation with the infarct volume at inclusion. In fact, a limitation of our study is related to the fact that the measured volume matches the ischemic core and does not include the volume of potentially reversible ischemia (penumbra). It has been suggested that inflammatory processes take place in the penumbral area and contribute to potential enlargement of the infarct. ${ }^{32}$ Furthermore, the measured volumes on diffusion do not match with the final volume, especially in treated patients. ${ }^{33,34}$ A potential association between neutrophil abnormalities and the progression of damage in the penumbra and the final ischemic volume have to be explored in further studies. In addition, we had a relatively limited number of 
patients, and our observations must be corroborated in larger cohorts.

Despite neutrophil hyperactivation, intravascular NETosis decreased in patients with IS starting at D2 after IS. This discrepancy with previous data ${ }^{35}$ might be related to the different techniques used to study NETosis. As chromatin constitutes the backbone of NETs, and MPO is present in the NETs, we adopted complexes of nucleosomes and MPO as the definition of NET remnants, as suggested, ${ }^{17}$ and quantified serum NET levels by detecting the MPO-DNA complexes. The mixing of the nuclear components with the content of azurophilic granules is a critical step in NETosis and enables the formation of complexes between nucleosomes and MPO. In contrast, in a previous study performed in patients with IS, ${ }^{35} 3$ markers were evaluated: plasma cell-free DNA, nucleosomes, and citrullination of histone 3, all only indirect markers of NETs. The decrease in intravascular NETosis observed in our study could be due to the decreased capacity of stimulated PMNs to produce NETs ${ }^{11}$ and to the presence of NETs in thrombotic occlusions from patients with IS. ${ }^{36}$

The occurrence of neutrophil reverse transmigration after ischemia/reperfusion injury has previously been reported in animal models. ${ }^{23}$ Our findings describe an increased percentage of neutrophils with an rTEM (CD54 ${ }^{\text {high }}$ CXCR ${ }^{\text {dim }}$ ) phenotype in human IS. The recent report that rTEM neutrophils show an activated morphology with unaltered antimicrobial activity suggests that they might be deleterious if reinfiltrated into remote organs. ${ }^{37}$ Moreover, rTEM neutrophils are relatively rigid, a physical characteristic that might delay their passage through the tissue microvasculature and prolong contact with the sinusoids. These cells could become mechanically entrapped in the microvasculature of major organs (most likely in the pulmonary circulation). We thus postulate that a larger population of functionally primed, recirculating neutrophils in patients with IS, with a predilection for mechanical or adhesive entrapment in the vasculature, could contribute to distant organ damage and multiorgan failure. As these rTEM neutrophils have been characterized by their prolonged survival and enhanced oxidative burst, ${ }^{38}$ their expansion in patients with IS might explain, at least in part, the higher ROS production and the lower rate of neutrophil apoptosis in our cohort. Furthermore, because rTEM neutrophils are reported to inhibit T-cell proliferation, ${ }^{9}$ we can speculate that they might contribute to the $\mathrm{T}$ lymphopenia observed during the stroke-induced immunodepression phase.

Consistent with data suggesting that sJAM-C may serve as an indirect marker of neutrophil rTEM, ${ }^{23,38}$ higher levels of this adhesion molecule in patients with IS associated with the percentage of rTEM neutrophils. On the other hand, sJAM-C also acts as a potent proangiogenic molecule with chemotactic action for endothelial cells, and it induces their formation of vascular tubes in vitro. ${ }^{39}$ After ischemic injury, angiogenesis increases the number of new collateral conduits, enhancing the blood supply and improving ischemic brain function. ${ }^{40}$ Despite this potential beneficial action of sJAM-C, we clearly observed that the sJAM-C level was higher in the severe stroke group.

As previously reported, ${ }^{41,42}$ we observed higher circulating levels of the proinflammatory cytokine IL-18 and of the DAMP HMGB-1 in patients with IS than in age-matched HCs. IL-18 production requires activation of the inflammasome, a group of multimeric protein complexes that sense stress signals, activate caspase-1, and thus cause its proteolytic cleavage. Notably, IS increases the expression and activation of the Nod-like receptor (NLR) pyrin domain containing 1 and 3 (NLRP1 and NLRP3) inflammasome proteins and both IL-1 $\beta$ and IL-18 in neurons. ${ }^{43}$ IL- $18^{44}$ and HMGB1 ${ }^{26}$ enhance neutrophil functions, including priming neutrophils for oxidative burst and degranulation. In turn, activated neutrophils produce IL-18. In addition, necrotic cells, including necrotic neutrophils, passively release HMGB1. Of interest, we found that the percentage of necrotic neutrophils was higher in patients with IS than HCs. These processes together might thus maintain a vicious cycle. We also observed that increased plasma levels of HMGB-1 were associated with markers of neutrophil activation.

The phenotypic and functional neutrophil changes that accompany acute IS may also be related to the expansion of the overactive aged neutrophil subpopulation. A recent study showed that neutrophil aging is regulated by the microbiota. ${ }^{8}$ Recent studies report gut dysbiosis in patients with IS. ${ }^{45,46}$ Commensal microbiota affects IS outcome in mice with ischemic brain injury by impairing immune homeostasis in the small intestine, thus increasing IL- $17^{+} \gamma \delta \mathrm{T}$ cells and decreasing regulatory $\mathrm{T}$ cells (Tregs). ${ }^{47}$ Of note, $\gamma \delta \mathrm{T}$ cells, a major lymphocyte population with innate immune features, can aggravate ischemic brain injury by secreting $\mathrm{IL}-17$ and generating chemotactic signals for peripheral myeloid cells such as neutrophils. ${ }^{48}$ In contrast, Tregs are known to inhibit neutrophil functions ${ }^{49}$ : in rodent IS models, adoptively transferred Tregs exert a protective effect by modulating peripheral neutrophils and thus prevent proteolytic damage to the $\mathrm{BBB} .{ }^{50}$ Alterations in the microbiota could contribute both to expansion of senescent neutrophils and changes in neutrophil activation in patients with IS. We can speculate that modulation of the microbiome might affect disease progression by limiting neutrophil aging and reduction production of neutrophil-derived cytotoxic molecules.

We demonstrated that homeostasis of circulating neutrophils is impaired during acute IS. These findings need to be replicated in an independent cohort of patients in which a meticulous definition of neutrophil subsets will be performed. The expansion of aged neutrophils might play a role in poststroke systemic inflammation and BBB disruption, and rTEM neutrophils might further contribute to the propagation of systemic inflammation. Our findings highlight new potential 
therapeutic approaches of stroke based on neutrophil modulation, in particular, by rebalancing the ratio of senescent to immunosuppressive neutrophils or decreasing reverse neutrophil transmigration or both. Further studies are needed, however, to determine whether these changes might serve as a predictive indicator of IS.

\section{Author contributions}

C. Elbim conceived the study, designed the experiments, and supervised the project. D. Weisenburger-Lile and Y. Dong conducted the experiments and analyzed the data. D. Weisenburger-Lile, M. Yger, G.F. Polara, and B. Lapergue were involved in patient recruitment and characterization and in the data analysis. G. Weisenburger-Lile, T. Chaigneau, and R. Zapata Ochoa participated in the data analysis. D. Weisenburger-Lile, S. Alamowitch, and C. Elbim wrote the manuscript. All authors read and approved the content of the final manuscript.

\section{Acknowledgment}

The authors thank the nursing staff of the Stroke Unit of Saint-Antoine Hospital Pitié Salpêtrière Hospital and Foch Hospital for the patient management. The authors gratefully acknowledge Annie Munier from the LUMIC flow cytometry facility. DWL received a grant from the "Société Francaise de Neurologie" for a part of this work.

\section{Study funding}

No targeted funding reported.

\section{Disclosure}

The authors report no disclosures relevant to the manuscript. Disclosures available: Neurology.org/NN.

\section{Publication history}

Received by Neurology: Neuroimmunology \& Neuroinflammation December 19, 2018. Accepted in final form March 12, 2019.

\section{References}

1. Ross AM, Hurn P, Perrin N, Wood L, Carlini W, Potempa K. Evidence of the peripheral inflammatory response in patients with transient ischemic attack. J Stroke Cerebrovasc Dis 2007;16:203-207.

2. Neumann J, Riek-Burchardt M, Herz J, et al. Very-late-antigen-4 (VLA-4)-mediated brain invasion by neutrophils leads to interactions with microglia, increased ischemic injury and impaired behavior in experimental stroke. Acta Neuropathol 2015;129: 259-277.

3. Shi Y, Zhang L, Pu H, et al. Rapid endothelial cytoskeletal reorganization enables early blood-brain barrier disruption and long-term ischaemic reperfusion brain injury. Nat Commun 2016;7:10523.

4. Perez-de-Puig I, Miro-Mur F, Ferrer-Ferrer M, et al. Neutrophil recruitment to the brain in mouse and human ischemic stroke. Acta Neuropathol 2015;129:239-257.

5. Enlimomab Acute Stroke Trial I. Use of anti-ICAM-1 therapy in ischemic stroke: results of the Enlimomab Acute Stroke Trial. Neurology 2001;57:1428-1434.

6. Krams M, Lees KR, Hacke W, et al. Acute stroke therapy by inhibition of neutrophils (ASTIN): an adaptive dose-response study of UK-279,276 in acute ischemic stroke. Stroke 2003;34:2543-2548.

7. Gunzer M. Traps and hyper inflammation-new ways that neutrophils promote or hinder survival. Br J Haematol 2014;164:189-199.

8. Zhang D, Chen G, Manwani D, et al. Neutrophil ageing is regulated by the microbiome. Nature 2015;525:528-532.

9. Pillay J, Kamp VM, van Hoffen E, et al. A subset of neutrophils in human systemic inflammation inhibits T cell responses through Mac-1. J Clin Invest 2012;122:327-336.

10. de Oliveira S, Rosowski EE, Huttenlocher A. Neutrophil migration in infection and wound repair: going forward in reverse. Nat Rev Immunol 2016;16:378-391.
11. Ruhnau J, Schulze K, Gaida B, et al. Stroke alters respiratory burst in neutrophils and monocytes. Stroke 2014;45:794-800.

12. Kelly PJ, Morrow JD, Ning M, et al. Oxidative stress and matrix metalloproteinase-9 in acute ischemic stroke: the biomarker evaluation for antioxidant therapies in stroke (BEAT-Stroke) study. Stroke 2008;39:100-104.

13. Pallister I, Bhatia R, Katpalli G, Allison D, Parker C, Topley N. Alteration of polymorphonuclear neutrophil surface receptor expression and migratory activity after isolation: comparison of whole blood and isolated PMN preparations from normal and postfracture trauma patients. J Trauma 2006;60:844-850.

14. Furie KL, Jayaraman MV. 2018 guidelines for the early management of patients with acute ischemic stroke. Stroke 2018;49:509-510.

15. Campillo-Gimenez L, Casulli S, Dudoit Y, et al. Neutrophils in antiretroviral therapycontrolled HIV demonstrate hyperactivation associated with a specific IL-17/IL-22 environment. J Allergy Clin Immunol 2014;134:1142-1152.e5.

16. Dong Y, Lagarde J, Xicota L, et al. Neutrophil hyperactivation correlates with Alzheimer's disease progression. Ann Neurol 2018;83:387-405.

17. Kessenbrock K, Krumbholz M, Schonermarck U, et al. Netting neutrophils in autoimmune small-vessel vasculitis. Nat Med 2009;15:623-625.

18. Bejot Y, Bailly H, Durier J, Giroud M. Epidemiology of stroke in Europe and trends for the 21st century. Presse Med 2016;45:e391-e398.

19. Xue J, Huang W, Chen X, et al. Neutrophil-to-Lymphocyte ratio is a prognostic marker in acute ischemic stroke. J Stroke Cerebrovasc Dis 2017;26:650-657.

20. Vogelgesang A, Lange C, Blumke L, et al. Ischaemic stroke and the recanalization drug tissue plasminogen activator interfere with antibacterial phagocyte function. J Neuroinflammation 2017;14:140.

21. Martinod K, Wagner DD. Thrombosis: tangled up in NETs. Blood 2014;123: 2768-2776.

22. Sauce D, Dong Y, Campillo-Gimenez L, et al. Reduced oxidative burst by primed neutrophils in the elderly individuals is associated with increased levels of the CD16bright/CD62Ldim immunosuppressive subset. J Gerontol A Biol Sci Med Sci 2017;72:163-172.

23. Colom B, Bodkin JV, Beyrau M, et al. Leukotriene B4-neutrophil elastase Axis drives neutrophil reverse transendothelial cell migration in vivo. Immunity 2015;42: 1075-1086.

24. Shichita T, Ito M, Yoshimura A. Post-ischemic inflammation regulates neural damage and protection. Front Cell Neurosci 2014;8:319.

25. Elbim C, Bailly S, Chollet-Martin S, Hakim J, Gougerot-Pocidalo MA. Differential priming effects of proinflammatory cytokines on human neutrophil oxidative burst in response to bacterial N-formyl peptides. Infect Immun 1994;62:2195-2201.

26. Berthelot F, Fattoum L, Casulli S, Gozlan J, Marechal V, Elbim C. The effect of HMGB1, a damage-associated molecular pattern molecule, on polymorphonuclear neutrophil migration depends on its concentration. J Innate Immun 2012;4:41-58.

27. Brouns R, Wauters A, De Surgeloose D, Marien P, De Deyn PP. Biochemical markers for blood-brain barrier dysfunction in acute ischemic stroke correlate with evolution and outcome. Eur Neurol 2011;65:23-31.

28. Ullen A, Singewald E, Konya V, et al. Myeloperoxidase-derived oxidants induce bloodbrain barrier dysfunction in vitro and in vivo. PLoS One 2013;8:e64034.

29. Gidday JM, Gasche YG, Copin JC, et al. Leukocyte-derived matrix metalloproteinase9 mediates blood-brain barrier breakdown and is proinflammatory after transient focal cerebral ischemia. Am J Physiol Heart Circ Physiol 2005;289:H558-H568.

30. Turner RJ, Sharp FR. Implications of MMP9 for blood brain barrier disruption and hemorrhagic transformation following ischemic stroke. Front Cell Neurosci 2016;10:56.

31. Uhl B, Vadlau Y, Zuchtriegel G, et al. Aged neutrophils contribute to the first line of defense in the acute inflammatory response. Blood 2016;128:2327-2337.

32. Vidale S, Consoli A, Arnaboldi M, Consoli D. Postischemic inflammation in acute stroke. J Clin Neurol 2017;13:1-9.

33. Luby M, Warach SJ, Nadareishvili Z, Merino JG. Immediate changes in stroke lesion volumes post thrombolysis predict clinical outcome. Stroke 2014;45:3275-3279.

34. Kate MP, Riaz P, Gioia L, et al. Dynamic evolution of diffusion-weighted imaging lesions in patients with minor ischemic stroke. Stroke 2015;46:2318-2321.

35. Valles J, Lago A, Santos MT, et al. Neutrophil extracellular traps are increased in patients with acute ischemic stroke: prognostic significance. Thromb Haemost 2017; 117:1919-1929.

36. Ducroux C, Di Meglio L, Loyau S, et al. Thrombus neutrophil extracellular traps content impair tPA-induced thrombolysis in acute ischemic stroke. Stroke 2018;49:754-757.

37. Ellett F, Elks PM, Robertson AL, Ogryzko NV, Renshaw SA. Defining the phenotype of neutrophils following reverse migration in zebrafish. J Leukoc Biol 2015;98:975-981.

38. Buckley CD, Ross EA, McGettrick HM, et al. Identification of a phenotypically and functionally distinct population of long-lived neutrophils in a model of reverse endothelial migration. J Leukoc Biol 2006;79:303-311.

39. Rabquer BJ, Amin MA, Teegala N, et al. Junctional adhesion molecule-C is a soluble mediator of angiogenesis. J Immunol 2010;185:1777-1785.

40. Fan Y, Yang GY. Therapeutic angiogenesis for brain ischemia: a brief review. J Neuroimmune Pharmacol 2007;2:284-289.

41. Ormstad H, Aass HC, Lund-Sorensen N, Amthor KF, Sandvik L. Serum levels of cytokines and C-reactive protein in acute ischemic stroke patients, and their relationship to stroke lateralization, type, and infarct volume. J Neurol 2011;258: $677-685$.

42. Tsukagawa $T$, Katsumata R, Fujita M, et al. Elevated serum high-mobility group box-1 protein level is associated with poor functional outcome in ischemic stroke. J Stroke Cerebrovasc Dis 2017;26:2404-2411. 
43. Fann DY, Lim YA, Cheng YL, et al. Evidence that NF-kappaB and MAPK signaling promotes NLRP inflammasome activation in neurons following ischemic stroke. Mol Neurobiol 2018;55:1082-1096.

44. Elbim C, Guichard C, Dang PM, et al. Interleukin-18 primes the oxidative burst of neutrophils in response to formyl-peptides: role of cytochrome b558 translocation and $\mathrm{N}$-formyl peptide receptor endocytosis. Clin Diagn Lab Immunol 2005;12:436-446.

45. Singh V, Roth S, Llovera G, et al. Microbiota dysbiosis controls the neuroinflammatory response after stroke. J Neurosci 2016;36:7428-7440.

46. Yamashiro K, Tanaka R, Urabe T, et al. Gut dysbiosis is associated with metabolism and systemic inflammation in patients with ischemic stroke. PLoS One 2017;12: e0171521.
47. Benakis C, Brea D, Caballero S, et al. Commensal microbiota affects ischemic stroke outcome by regulating intestinal gammadelta T cells. Nat Med 2016;22: $516-523$.

48. Gelderblom M, Weymar A, Bernreuther C, et al. Neutralization of the IL-17 axis diminishes neutrophil invasion and protects from ischemic stroke. Blood 2012;120: 3793-3802.

49. Lewkowicz P, Lewkowicz N, Sasiak A, Tchorzewski H. Lipopolysaccharide-activated $\mathrm{CD} 4+\mathrm{CD} 25+\mathrm{T}$ regulatory cells inhibit neutrophil function and promote their apoptosis and death. J Immunol 2006;177:7155-7163.

50. Li P, Gan Y, Sun BL, et al. Adoptive regulatory T-cell therapy protects against cerebral ischemia. Ann Neurol 2013;74:458-471. 


\title{
Neurology \\ Neuroimmunology \& Neuroinflammation
}

\author{
Harmful neutrophil subsets in patients with ischemic stroke: Association with disease \\ severity \\ David Weisenburger-Lile, Yuan Dong, Marion Yger, et al. \\ Neurol Neuroimmunol Neuroinflamm 2019;6; \\ DOI 10.1212/NXI.0000000000000571
}

This information is current as of May 15, 2019

\begin{abstract}
Updated Information \&
Services

References

Citations

Subspecialty Collections

Permissions \& Licensing

Reprints

including high resolution figures, can be found at: http://nn.neurology.org/content/6/4/e571.full.html

This article cites 50 articles, 14 of which you can access for free at: http://nn.neurology.org/content/6/4/e571.full.html\#\#ref-list-1

This article has been cited by 1 HighWire-hosted articles: http://nn.neurology.org/content/6/4/e571.full.html\#\#otherarticles

This article, along with others on similar topics, appears in the following collection(s):

All Immunology

http://nn.neurology.org//cgi/collection/all_immunology

Infarction

http://nn.neurology.org//cgi/collection/infarction its entirety can be found online at:

http://nn.neurology.org/misc/about.xhtml\#permissions

Information about ordering reprints can be found online:

http://nn.neurology.org/misc/addir.xhtml\#reprintsus
\end{abstract}

Information about reproducing this article in parts (figures,tables) or in

Neurol Neuroimmunol Neuroinflamm is an official journal of the American Academy of Neurology.

Published since April 2014, it is an open-access, online-only, continuous publication journal. Copyright

Copyright $@ 2019$ The Author(s). Published by Wolters Kluwer Health, Inc. on behalf of the American

Academy of Neurology.. All rights reserved. Online ISSN: 2332-7812.

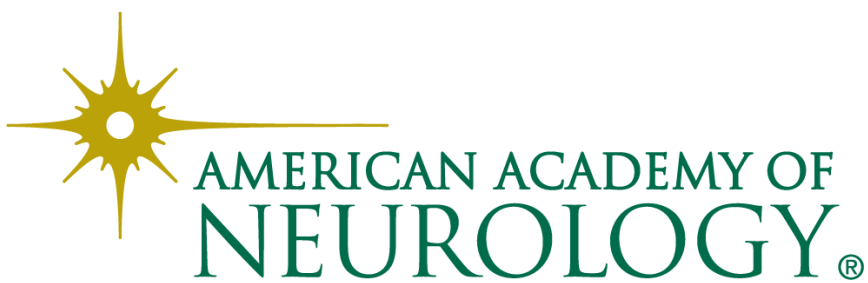

